\title{
Cerebral Perfusion and the Burden of Small Vessel Disease in Patients Referred to a Memory Clinic
}

\author{
Laurien Onkenhout ${ }^{a} \quad$ Nadine Appelmans ${ }^{a} \quad$ L. Jaap Kappelle ${ }^{a}$ Dineke Koek ${ }^{a}$ \\ Lieza Exalto $^{a}$ Jeroen de Bresser ${ }^{b}$ Geert Jan Biessels ${ }^{a}$ on behalf of the Utrecht \\ VCI Study Group \\ ${ }^{a}$ Department of Neurology and Neurosurgery, Brain Center Rudolf Magnus, University Medical Center Utrecht, \\ Utrecht, The Netherlands; ${ }^{b}$ Department of Radiology, Leiden University Medical Center, Leiden, The Netherlands
}

\section{Keywords \\ Cerebral perfusion - Small vessel disease - White matter hyperintesities · Lacunes · Microbleeds · Enlarged perivascular spaces}

\begin{abstract}
Background: Cerebral small vessel disease (SVD) lesions on $\mathrm{MRI}$ are common in patients with cognitive impairment. It has been suggested that cerebral hypoperfusion is involved in the etiology of these lesions. Objective: The aim of the study was to determine the relationship between cerebral blood flow (CBF) and SVD burden in patients referred to a memory clinic with SVD on MRI. Method: We included 132 memory clinic patients (mean age $73 \pm 10,56 \%$ male) with SVD on MRI. We excluded patients with large non-lacunar cortical infarcts. Global CBF (mL/min per $100 \mathrm{~mL}$ of brain tissue) was derived from 2-dimensional phase-contrast MRI focused on the internal carotid arteries and the basilar artery. SVD burden was defined as the sum of (each 1 point): white matter hyperintensities (WMHs) Fazekas 1 or more, lacunes, microbleeds (MBs), or enlarged perivascular spaces (PVS) presence, and each SVD feature separately. Linear regression analyses were performed to study the association between CBF and SVD burden, age- and sex-adjusted. Results: Medi-
\end{abstract}

\begin{tabular}{|c|c|}
\hline $\begin{array}{l}\text { karger@karger.com } \\
\text { www.karger.com/ced }\end{array}$ & $\begin{array}{l}\text { (C) } 2020 \text { The Author(s) } \\
\text { Published by S. Karger AG, Basel }\end{array}$ \\
\hline OPEN A & $\begin{array}{l}\text { This article is licensed under the Creative Commons Attribution- } \\
\text { NonCommercial-NoDerivatives } 4.0 \text { International License (CC BY- } \\
\text { NC-ND) (http://www.karger.com/Services/OpenAccessLicense). } \\
\text { Usage and distribution for commercial purposes as well as any dis- } \\
\text { tribution of modified material requires written permission. }\end{array}$ \\
\hline
\end{tabular}

an SVD burden score was 2, 36.4\% of patients had MBs, $35.6 \%$ lacunar infarcts, $48.4 \%$ intermediate to severe enlarged PVS, and $57.6 \%$ a WMH Fazekas score 2 or more. Median WMH volume was $21.4 \mathrm{~mL}$ ( $25 \%$ quartile: $9.6 \mathrm{~mL}, 75 \%$ quartile: $32.5 \mathrm{~mL}$ ). Mean CBF \pm SD was $44.0 \pm 11.9 \mathrm{~mL} / \mathrm{min}$ per $100 \mathrm{~mL}$ brain. There was no relation between CBF and overall SVD burden (CBF difference per burden score point [95\% Cl]: $-0.5[-2.4 ; 1.4] \mathrm{mL} / \mathrm{min} / 100 \mathrm{~mL}$ brain, $p=0.9)$. CBF did also not differ according to presence or absence or an high burden of any of the individual SVD features. Moreover, there was no significant relation between WMH volume and CBF (CBF difference per $\mathrm{ml}$ increase in WMH [95\% Cl] -0.6 $[-1.5 ; 0.3] \mathrm{mL} / \mathrm{min} / 100 \mathrm{~mL}$ brain $p=0.2)$. Conclusion: Global CBF was not related to overall SVD burden or with individual SVD features in this memory clinic cohort, indicating that in this setting these lesions were not primarily due to cerebral hypoperfusion.

(C) 2020 The Author(s)

Published by S. Karger AG, Basel

\section{Introduction}

Cerebral small vessel disease (SVD) is particularly common in older individuals. It refers to a group of pathogenic processes with possibly different etiologies

Laurien Onkenhout

Department of Neurology and Neurosurgery, University Medical Center Utrecht Heidelberglaan 100

NL-3508 GA Utrecht (The Netherlands)

l.p.onkenhout@umcutrecht.nl 
that affect the small vessels of the brain. It can manifest itself on MRI as lacunes, microbleeds (MBs), white matter hyperintensities (WMHs), and enlarged perivascular spaces (PVS). SVD burden on MRI is associated with an increased risk of dementia and stroke.

The processes that lead to SVD lesions are still largely unknown, but it is thought that chronic cerebral hypoperfusion may play a role. A systematic review has suggested a relationship between WMHs and perfusion [1]. The relationship between perfusion and other features of SVD has been explored in only a few studies. In the current study, we hypothesized that global alterations in the small vessels throughout the brain could be associated with global perfusion changes. We examined if overall SVD burden is related to cerebral blood flow (CBF). Since different MRI features of SVD may reflect different disease processes, the relationship between the individual features of SVD and CBF was also explored. We conducted our study in memory clinic patients with SVD of various severities.

\section{Method}

\section{Population}

Patients were selected from the vascular cognitive impairment cohort of the University Medical Center Utrecht [2]. This cohort consists of consecutive patients with evidence of vascular brain injury on MRI, who attended the memory clinic between 2009 and 2013 [3]. All patients with at least a minimum burden of SVD were selected. Presence of SVD was defined as WMH Fazekas scale grade 1 or more lacune(s); 1 or more cerebral MB(s) and/or moderately to severely enlarged PVS (grade 2-4) [4]. Patients with evidence of co-existing neurodegenerative disorders (such as Alzheimer's dementia) were included in the cohort, in line with proposed VCI criteria [2]. Patients with a large (sub)cortical infarct on MRI (with a volume of $3 \mathrm{~mL}$ or more) were excluded because these large infarcts might confound the relation between CBF and SVD. Patients with primary nonvascular/non-degenerative causes of cognitive dysfunction (e.g., brain tumors and traumatic brain injury) or psychiatric disease (other than depression) and patients with monogenetic (non-) vascular (e.g., NOTCH3) causes for cognitive dysfunction were excluded.

This study was approved by the institutional review board of the University Medical Center Utrecht, The Netherlands. All included patients provided written informed consent.

\section{MRI Protocol and Visual Assessment of SVD Features}

MRI scans of the brain were performed on a Philips 3 T MRI scanner (Intera; Philips, Best, the Netherlands) with a standardized scan protocol that included 3D T1-weighted images (TR/TE: $7.2 / 2.9 \mathrm{~ms}$; reconstructed voxel size $\left.1.0 \times 1.0 \times 1.0 \mathrm{~mm}^{3}\right)$, $2 \mathrm{D} \mathrm{T} 2$ weighted turbo spin echo (TSE) images (TR/TE: 3,194/14 ms; reconstructed voxel size $0.96 \times 0.95 \times 3.0 \mathrm{~mm}^{3}$ ), T2*-weighted images (TR/TE: $1,653 / 20 \mathrm{~ms}$; reconstructed voxel size $0.96 \times 0.95 \times$ $3.0 \mathrm{~mm}^{3}$ ), fluid-attenuated inversion recovery (FLAIR) images (TR/TE/TI: $11,000 / 125 / 2,800 \mathrm{~ms}$; reconstructed voxel size $0.96 \times$ $\left.0.95 \times 3.0 \mathrm{~mm}^{3}\right)$. WMH burden was assessed by the Fazekas scale [5] and WMH volume. Both the presence and number of lacunes and MBs were rated according to the STRIVE criteria [6]. Enlarged PVS were rated based on the method used by Staals et al. [4]. Medial temporal lobe atrophy was assessed according to the Scheltens scale (scores for both hemispheres were averaged) [7].

Determining the total burden score of SVD based on MRI features was also performed in accordance with Staals et al. [4]. The total burden was rated on an ordinal scale from 0 to 4 . This was performed by adding up the presence of each of the 4 MRI features for SVD. A single point was awarded for the presence of each of these features; the sum of these features is the total burden score of SVD.

\section{MRI Image Processing}

Assessment of Brain Volume

Brain volumetric measurements were performed by automated segmentation using the Computational Anatomy Toolbox CAT12 [8]. The automated CAT12 segmentations for total brain and $\mathrm{WMH}$ volume were subsequently corrected for by hand segmentation of stroke lesion volume (e.g., lacunes, non-lacunar infarcts, and hemorrhages). An extended explanation of the semiautomated processing pipeline was previously published [9].

Measurements of CBF and Assessment of Relative Total Brain Perfusion

The CBF was calculated in the following way: a two-dimensional phase-contrast MRI slice was acquired at the level just below the skull base in which both the internal carotid arteries and the basilar artery were visible. Next, the regions of interest were manually segmented by drawing an ROI closely around the vessel lumens of both internal carotid arteries and the basilar artery on the phasedifference images. An automatic calculation program was used to calculate the flux. The CBF was calculated by summing up the 3 fluxes giving the total CBF. We then calculated the mean CBF by dividing the total $\mathrm{CBF}(\mathrm{mL} / \mathrm{min}$ ) by total brain volume (in $\mathrm{ml}$ ) and multiplying by 100 to obtain the $\mathrm{CBF}$ in $\mathrm{mL} / \mathrm{min}$ per $100 \mathrm{~mL}$ brain tissue [10]. With this approach, we achieved an excellent intraclass correlation coefficient (ICC), both for agreement between different raters $(\mathrm{ICC}=0.928)$ and within a single rater $(\mathrm{ICC}=$ $0.961)$.

\section{Data Analysis}

A linear regression model was applied to investigate the relationship between CBF and the SVD burden score. This model was also applied to investigate the relationship between $\mathrm{CBF}$ and the 4 individual SVD features (MBs, lacunes, WMH Fazekas and volume, and enlarged PVS). All analyses were corrected for age and sex. In our model, CBF was analyzed as the dependent variable, thus providing results in $\mathrm{mL} / \mathrm{min} / 100$ brain in relation to the SVD feature, making the data easier to interpret. Given the distribution of the different SVD features in our cohort, we were powered to detect effect sizes of around 0.4 and above (i.e., a CBF difference of around $5 \mathrm{~mL} / \mathrm{min} / 100 \mathrm{~mL}$ tissue) with a power 0.8 and alpha smaller than 0.05 in dichotomous analyses. We also performed a secondary logistic regression analysis with CBF as a determinant and dichotomized SVD features as a dependent variable. Sensitivity analyses were also performed to investigate the role of neurodegenerative diseases (such as Alzheimer's disease) in the analyses. All data analyses were carried out in IBM SPSS statistics (version 22 ), and a $p$ value $<0.05$ was considered statistically significant. 
Table 1. Demographics, diagnoses, and SVD burden of the study population

\begin{tabular}{|c|c|}
\hline & $\begin{array}{l}\text { Patients } \\
(N=132)\end{array}$ \\
\hline Age, years & $73 \pm 10$ \\
\hline Male sex, $n(\%)$ & $74(56.1)$ \\
\hline \multicolumn{2}{|l|}{ Diagnoses, n (\%) } \\
\hline No objective cognitive impairment & $22(16.7)$ \\
\hline Mild cognitive impairment & $44(33.3)$ \\
\hline Dementia & $66(50.0)$ \\
\hline $\begin{array}{l}\text { Thereof possible or probable Alzheimer's } \\
\text { disease }\end{array}$ & $53(40.2)$ \\
\hline $\mathrm{CBF}[\mathrm{mL} / \mathrm{min} / 100 \mathrm{~mL}$ brain $]$ & $44.0 \pm 11.9$ \\
\hline \multicolumn{2}{|l|}{ Neuroimaging features } \\
\hline \multicolumn{2}{|l|}{ MBs, $n(\%)$} \\
\hline None & $84(63.6)$ \\
\hline Any (1 or more) & $48(36.4)$ \\
\hline Thereof 1 & $19(14.4)$ \\
\hline Thereof multiple & $29(21.9)$ \\
\hline \multicolumn{2}{|l|}{ Lacunar infarcts, $n(\%)$} \\
\hline None & $85(64.4)$ \\
\hline Any ( 1 or more) & $47(35.6)$ \\
\hline Thereof 1 & $16(12.1)$ \\
\hline Thereof multiple & $31(23.5)$ \\
\hline WMH volume, $\mathrm{mL}$ & $21.4[9.6 ; 32.5]$ \\
\hline \multicolumn{2}{|l|}{ WMHs, $n(\%)$} \\
\hline Fazekas 0 & $4(3.0)$ \\
\hline Fazekas 1 & $52(39.4)$ \\
\hline Fazekas 2 & $59(44.7)$ \\
\hline Fazekas 3 & $17(12.9)$ \\
\hline \multicolumn{2}{|l|}{ Enlarged PVS, $n(\%)$} \\
\hline Score 0 & 0 \\
\hline Score 1 (mild) & $4(3.2)$ \\
\hline Score 2 (moderate) & $61(48.4)$ \\
\hline Score 3 (intermediate) & $60(47.6)$ \\
\hline Score 4 (severe) & $1(0.8)$ \\
\hline
\end{tabular}

Data are presented as mean \pm SD, $n(\%)$, or median $[25 \%$ quartile; $75 \%$ quartile]. Throughout the table, all percentages are relative to the sample size of 132 , except for PVS, ratings that were available from 126 participants. SVD, small vessel disease; CBF, cerebral blood flow; $\mathrm{WMH}$, white matter hyperintensities; MBs, microbleeds; PVS, perivascular spaces.

\section{Results}

\section{Demographics SVD Features and CBF}

From the 196 patients in the cohort, 21 were excluded from the present study for presence of large cortical brain infarcts. Additionally, we excluded 25 patients for lack of a CBF MRI sequence and 18 for a CBF sequence or brain volumetry of insufficient quality. The 132 included pa-
Table 2. CBF according to total SVD burden score

\begin{tabular}{llll}
\hline $\begin{array}{l}\text { SVD burden score } \\
{[0-4]}\end{array}$ & $\begin{array}{l}\text { Age, } \\
\text { years }\end{array}$ & $\begin{array}{l}\text { Male, } \\
\%\end{array}$ & $\begin{array}{l}\text { CBF mL/min per } \\
100 \mathrm{~mL} \text { brain }\end{array}$ \\
\hline $0(n=9,6.8 \%)$ & $67 \pm 10$ & 56 & $43.1 \pm 15.9$ \\
$1(n=32,24.2 \%)$ & $70 \pm 8$ & 53 & $45.4 \pm 11.8$ \\
$2(n=47,35.6 \%)$ & $73 \pm 10$ & 57 & $44.8 \pm 11.0$ \\
$3(n=29,22.0 \%)$ & $76 \pm 10$ & 64 & $39.9 \pm 12.8$ \\
$4(n=15,11.4 \%)$ & $73 \pm 13$ & 60 & $46.2 \pm 11.7$ \\
\hline
\end{tabular}

Data on age are presented as mean $\pm \mathrm{SD}$, data on $\mathrm{CBF}$ are presented as unadjusted means \pm SD for interpretation purposes; results of age- and sex-adjusted statistical models are presented in the main text. SVD, burden score based on all SVD; 4 MRI, features with minimum score 0 and maximum 4 . SVD, small vessel disease; CBF, cerebral blood flow.

tients (56\% male; mean age 73 years) had a median SVD burden score of 2 , range minimum 0 , maximum $4 ; 36.4 \%$ of patients had 1 or more MBs, $35.6 \%$ had lacunes, and WMH Fazekas score of 1 or more was present in $97.0 \%$ of the study population in which $57.6 \%$ had a Fazekas score of 2 or more. WMH Fazekas score had a median of $2(25 \%$ quartile: $1,75 \%$ quartile: 2 ). The median $\mathrm{WMH}$ volume was $21.4 \mathrm{~mL}$ (25\% quartile: $9.6 \mathrm{~mL}, 75 \%$ quartile: 32.5 $\mathrm{mL})$. Of 126 participants with available perivascular space ratings, $48.4 \%$ had intermediate/severe enlarged PVS. Mean CBF \pm SD was $44.0 \pm 11.9 \mathrm{~mL} / \mathrm{min}$ per $100 \mathrm{~mL}$. Baseline characteristics are summarized in Table 1.

\section{Association between CBF and SVD Burden and Individual SVD Features}

CBF did not differ significantly according to overall SVD burden score (age- and sex-adjusted CBF difference per burden score point $[95 \% \mathrm{CI}]:-0.5[-2.4 ; 1.4] \mathrm{mL} /$ $\mathrm{min} / 100 \mathrm{~mL}$ brain, $p=0.9$; unadjusted CBF data are presented in Table 2). Furthermore, there was no association between individual SVD features and CBF (unadjusted CBF data are presented in Table 3). There was no difference in CBF between patients without and with any MBs (age- and sex-adjusted CBF difference [95\% CI]: 0.3 $[-4.0 ; 4.3] \mathrm{mL} / \mathrm{min} / 100 \mathrm{~mL}$ brain, $p=1.0$; in those with any $\mathrm{MBs}$, the average $\mathrm{MB}$ count $\pm \mathrm{SD}$ was $4 \pm 5$ ), or between patients without any and those with multiple MBs (adjusted CBF difference [95\% CI]: $-1.0[-5.9 ; 4.0] \mathrm{mL} /$ $\mathrm{min} / 100 \mathrm{~mL}$ brain $p=0.7$; in those with multiple MBs, the average MB count \pm SD was $6 \pm 5$ ). CBF was similar in patients without and with any lacunar infarcts (adjusted CBF difference $[95 \% \mathrm{CI}]:-0.5[-4.6 ; 3.5] \mathrm{mL} / \mathrm{min} / 100$ $\mathrm{mL}$ brain, $p=0.8$; in those with any lacunes, the average 
Table 3. CBF according to individual SVD features

\begin{tabular}{|c|c|c|c|}
\hline SVD feature & $\begin{array}{l}\text { Age, } \\
\text { years }\end{array}$ & $\begin{array}{l}\text { Male, } \\
\%\end{array}$ & $\begin{array}{l}\mathrm{CBF} \mathrm{mL} / \mathrm{min} \\
\text { per } 100 \mathrm{~mL} \\
\text { brain }\end{array}$ \\
\hline \multicolumn{4}{|l|}{$\operatorname{MBs}[n=132]$} \\
\hline None $[n=84]$ & $72 \pm 9$ & 60 & $44.1 \pm 12.2$ \\
\hline Any ( 1 or more) $[n=48]$ & $74 \pm 12$ & 48 & $43.8 \pm 12.2$ \\
\hline Thereof $1[n=19]$ & $74 \pm 12$ & 45 & $44.7 \pm 11.0$ \\
\hline Thereof multiple [ $n=29$ ] & $74 \pm 12$ & 60 & $42.0 \pm 13.2$ \\
\hline \multicolumn{4}{|l|}{ Lacunar infarcts $[n=132]$} \\
\hline None $[n=85]$ & $73 \pm 10$ & 49 & $44.2 \pm 12.2$ \\
\hline Any (1 or more) $[n=47]$ & $74 \pm 11$ & 68 & $43.5 \pm 11.4$ \\
\hline Thereof $1[n=16]$ & $74 \pm 11$ & 56 & $43.3 \pm 10.2$ \\
\hline Thereof multiple $[n=31]$ & $72 \pm 10$ & 74 & $44.6 \pm 12.5$ \\
\hline \multicolumn{4}{|l|}{ WMH Fazekas $[n=132]$} \\
\hline Fazekas $0[n=4]$ & $64 \pm 11$ & 50 & $46.5 \pm 4.7$ \\
\hline Fazekas $1[n=52]$ & $70 \pm 10$ & 64 & $45.6 \pm 12.7$ \\
\hline Fazekas $2[n=59]$ & $75 \pm 10$ & 58 & $43.4 \pm 12.2$ \\
\hline Fazekas $3[n=17]$ & $75 \pm 11$ & 59 & $39.8 \pm 10.3$ \\
\hline \multicolumn{4}{|l|}{ Enlarged PVS $[n=126]$} \\
\hline Score $0[n=0]$ & na & na & na \\
\hline Score 1 (mild) $[n=4]$ & $68 \pm 7$ & 75 & $46.4 \pm 10.1$ \\
\hline Score 2 (moderate) $[n=61]$ & $72 \pm 11$ & 51 & $46.4 \pm 11.2$ \\
\hline Score 3 (intermediate) $[n=60]$ & $74 \pm 9$ & 60 & $42.0 \pm 11.1$ \\
\hline Score 4 (severe) $[n=1]$ & 78 & 100 & 29.1 \\
\hline
\end{tabular}

Data on age are presented as mean $\pm \mathrm{SD}$, data on $\mathrm{CBF}$ are presented as unadjusted means $\pm \mathrm{SD}$, for interpretation purposes; results of age- and sex-adjusted statistical models are presented in the main text. SVD, small vessel disease; WMH, white matter hyperintensities; CBF, cerebral blood flow; MBs, microbleeds; PVS, perivascular spaces.

lacune count $\pm \mathrm{SD}$ was $4 \pm 3$ ), also in those with multiple and those without any infarcts (adjusted CBF difference [95\% CI]: $0.5[-4.3 ; 5.4] \mathrm{mL} / \mathrm{min} / 100 \mathrm{~mL}$ brain, $p=0.8$; in those with multiple lacunes, the average lacune count \pm SD was $5 \pm 3$ ). Moreover, $\mathrm{CBF}$ was similar in those with no/mild Fazekas WMH (Fazekas score $0 ; 1, n=56$, mean CBF \pm SD: $45.7 \pm 12.3 \mathrm{~mL} / \mathrm{min} / 100 \mathrm{~mL}$ brain) and those with moderate/severe Fazekas WMH (Fazekas score 2; 3, $n=76$, mean $\mathrm{CBF} \pm \mathrm{SD}: 42.8 \pm 11.5 \mathrm{~mL} / \mathrm{min} / 100 \mathrm{~mL}$ brain) (adjusted CBF difference [95\% CI]: $-0.6[-4.6 ; 3.4]$ $\mathrm{mL} / \mathrm{min} / 100 \mathrm{~mL}$ brain, $p=0.7)$. The actual WMH volume was also not significantly related to CBF (adjusted change in $\mathrm{CBF}$ per $\mathrm{ml}$ increase in WMHs $[95 \% \mathrm{CI}]-0.6[-1.5$; $0.3 \mathrm{~mL} / \mathrm{min} / 100 \mathrm{~mL}$ brain $p=0.2$ ). CBF did also not differ in those without and with intermediate/severe enlarged PVS (score 3; 4, $n=61$, mean CBF \pm SD: $41.8 \pm 11.1$ $\mathrm{mL} / \mathrm{min} / 100 \mathrm{~mL}$ brain) (adjusted CBF difference [95\%
$\mathrm{CI}]: 3.3[-7.1 ; 0.5] \mathrm{mL} / \mathrm{min} / 100 \mathrm{~mL}$ brain $p=0.1)$. Secondary logistic regression analyses with $\mathrm{CBF}$ as determinant, and the individual SVD features as a dependent variable provided largely similar results, although the relation between $\mathrm{CBF}$ and intermediate/severe PVS became statistically significant (OR [95\% CI]: 0.67 per $10 \mathrm{~mL} /$ $\min / 100 \mathrm{~mL}$ brain $[0.47 ; 0.95] p=0.03)$.

In order to rule out that our findings are driven by Alzheimer disease-related neurodegenerative pathology, we performed a sensitivity analysis excluding patients with a clinical diagnosis of dementia due to Alzheimer disease. These results were essentially similar compared to the main analysis of overall SVD burden (CBF difference per burden score point $[95 \% \mathrm{CI}] 0.3 \mathrm{~mL} / \mathrm{min} / 100 \mathrm{~mL}$ brain $[-1.9 ; 2.6] p=0.8)$.

\section{Discussion}

In this cohort of memory clinic patients with SVD features on MRI, in our primary analyses, neither overall SVD burden, nor individual MRI features were related to CBF. This study provides new perspectives on the relation between CBF and SVD [1] by assessing different MRI features of SVD. Previous studies on the relation between CBF and SVD have mainly focused on WMHs [1, 11-15]. A systematic review reported that $\mathrm{CBF}$ is lower in subjects with WMHs in a cross-sectional analysis [1], although this relation attenuated after age-matching. This is consistent with the tendency for a lower CBF with higher WMH burden that we observed in our study.

We found no relationship between $\mathrm{CBF}$ and MBs or lacunes after correction for age and sex in our primary or secondary analyses. A limited number of studies, both cross-sectional and longitudinal, have assessed the association between CBF and these features of SVD [5-13]. A single study on MBs, in a rather small and selected population of patients with symptomatic SVD, did show a relationship between multiple MBs ( 5 or more) and lower CBF in one of several brain regions tested [16]. With regard to lacunes, a study in a large cohort of patients with clinically manifested extra- or intracranial arterial disease observed no association between CBF and incident lesions [14]. Vice versa, baseline presence of lacunes did not predict change in $\mathrm{CBF}$ over time [14]. In our primary and secondary analyses, we found different results on the relationship between enlarged PVS and CBF. A single study on enlarged PVS, in a cohort of patients with minor stroke, observed no relation with CBF [17] in line with our primary findings. These differential results may be 
indicative of a weak relation between enlarged PVS and $\mathrm{CBF}$, that is, not consistently detected due to limited statistical power.

There are a number of possible explanations why we did not find an association between higher SVD burden and a lower global CBF. Although it may be reasonable to assume a linear relation between the burden of visible SVD lesions and global changes in the small vessels, such a relation is uncertain. More likely, our measure of global perfusion may not capture more subtle regional changes in the small vessels. An extreme example of a local $\mathrm{CBF}$ deficit causing an SVD lesion is a lacunar infarct. But also in and around WMH local changes in CBF can be found $[18,19]$. It has also been suggested that capillary flow disturbance due to SVD can lead to a less homogeneous blood flow, which can affect the oxygen extraction efficacy in tissue even if the overall CBF remains unchanged [20].

One of the strengths of our study is the substantial cohort of memory clinic patients with high-quality MRI data. A possible limitation of the study is that we obtained a measure of overall CBF at the level of the large extracranial arteries using two-dimensional phase-contrast MRI and did not measure $\mathrm{CBF}$ at the tissue level. Yet, the observed mean CBF values in our study are comparable to other studies that did use measures at the tissue level [113]. This does not exclude that there can be regional areas of abnormal perfusion at the site where lesions develop or have developed. Also, because all participants were selected for the presence of at least a minimal burden of SVDs, we could not compare CBF for patients with and without SVD. It might be argued that heterogeneity of our study population in terms of etiologies and co-morbidities, in particular Alzheimer's disease, is a limitation. Of note, we deliberately included patients from our memory clinic with different manifestations and burden of SVDs, to capture the full spectrum of SVDs in this setting. Moreover, post hoc stratified analyses by Alzheimer diagnosis (albeit without biomarker support) confirmed our main results. Other comorbidities could influence CBF. However, we excluded conditions that may affect CBF, in particular patients with large (sub)cortical infarct on MRI (with a volume similar or $>3 \mathrm{~mL}$ ), and none of the patients had an occlusion of the carotid arteries and/or basilar artery. Finally, in our population, given our statistical power, we were only able to detect relations with CBF of medium effect sizes. In conclusion, we found that in a memory clinic cohort, overall SVD burden and MRI features of SVD were not consistently related to CBF, indicating that in this setting, these lesions were not primarily due to cerebral hypoperfusion.

Cerebral Perfusion, Related to Small

Vessel Disease Burden?

\section{Acknowledgements}

The authors thank the members of the TRACE-VCI study group [2] supporting the present study.

\section{Statement of Ethics}

This study was conducted in compliance with the guidelines for human studies and in accordance with the World Medical Association Declaration of Helsinki. All subjects have given their written informed consent, and the study protocol was approved by the institutional review and ethics board of the University Medical Center Utrecht; study number TRACE-VCI 14-083.

\section{Conflict of Interest Statement}

Laurien Onkenhout, Nadine Appelmans, L. Jaap Kappelle, Dineke Koek, Lieza Exalto, Jeroen de Bresser, and Geert Jan Biessels report no disclosures.

\section{Funding Sources}

The TRACE-VCI study is supported by Vidi grant 917.11.384 and Vici grant 918.16.616 from ZonMw, The Netherlands, Organization for Health Research and Development, and grant 2010T073 from the Dutch Heart Association to Geert Jan Biessels.

\section{Author Contributions}

L.P. Onkenhout: study concept and design, interpretation of data, and drafting the manuscript. Nadine Appelmans: study concept and design, interpretation of data and CBF calculations. L. Jaap Kappelle: interpretation of data and critical revision of manuscript. Dineke Koek: interpretation of data and critical revision of manuscript. Lieza Exalto: data acquisition and critical revision of manuscript. Jeroen de Bresser: data acquisition, interpretation of data, and critical revision of manuscript. Geert Jan Biessels: study concept and design, supervision, obtaining funding, interpretation of data, and revising the manuscript for important intellectual content.

\section{References}

1 Shi Y, Thrippleton MJ, Makin SD, Marshall I, Geerlings MI, de Craen AJ, et al. Cerebral blood flow in small vessel disease: a systematic review and meta-analysis. J Cereb Blood Flow Metab. 2016;36(10):1653.

2 Boomsma JMF, Exalto LG, Barkhof F, van den Berg E, de Bresser J, Heinen R, et al. Vascular cognitive impairment in a memory clinic population: rationale and design of the "Utrecht-Amsterdam Clinical Features and Prognosis in Vascular Cognitive Impairment" (TRACE-VCI) Study. JMIR Res Protoc. $2017 ; 6(4): \mathrm{e} 60$. 
3 Ferro DA, Van Veluw SJ, Koek HL, Exalto LG, Biessels GJ. Cortical cerebral microinfarcts on 3 tesla MRI in patients with vascular cognitive impairment. J Alzheimers Dis. 2017;60(4): 1443-50.

4 Staals J, Makin SD, Doubal FN, Dennis MS, Wardlaw JM. Stroke subtype, vascular risk factors, and total MRI brain small-vessel disease burden. Neurology. 2014;83(14):122834.

5 Fazekas F, Chawluk JB, Alavi A, Hurtig HI, Zimmerman RA. MR signal abnormalities at $1.5 \mathrm{~T}$ in Alzheimer's dementia and normal aging. AJR Am J Roentgenol. 1987;149(2):3516.

6 Ferro DA, van Veluw SJ, Koek HL, Exalto LG, Biessels GJ. Utrecht vascular cognitive impairment (VCI) study group. Cortical cerebral microinfarcts on 3 tesla MRI in patients with vascular cognitive impairment. J Alzheimers Dis. 2017;60:1-8.

7 Ferro DA, van den Brink H, Exalto LG, Boomsma JMF, Barkhof F, Prins ND, et al. Clinical relevance of acute cerebral microinfarcts in vascular cognitive impairment. Neurology. 2019;92(14):e1558-66.

8 Gaser C, Dahnke R. CAT: a computational anatomy toolbox for the analysis of structural MRI data. 2016

9 Groeneveld ON, Moneti C, Heinen R, De Bresser J, Kuijf HJ, Exalto LG, et al. The clinical phenotype of vascular cognitive impairment in patients with type 2 diabetes mellitus. J Alzheimers Dis. 2019;68(1):311-22.
10 Tiehuis AM, Vincken KL, van den Berg E, Hendrikse J, Manschot SM, Mali WP, et al. Cerebral perfusion in relation to cognitive function and type 2 diabetes. Diabetologia. 2008;51(7):1321-6.

11 van Es AC, van der Grond J, ten Dam VH, de Craen AJ, Blauw GJ, Westendorp RG, et al. Associations between total cerebral blood flow and age related changes of the brain. PLoS One. 2010;5(3):e9825-6.

12 Kitagawa K, Oku N, Kimura Y, Yagita Y, Sakaguchi M, Hatazawa J, et al. Relationship between cerebral blood flow and later cognitive decline in hypertensive patients with cerebral small vessel disease. Hypertens Res. 2009; 32(9):816-20.

13 Appelman AP, van der Graaf Y, Vincken KL, Tiehuis AM, Witkamp TD, Mali WP, et al. Total cerebral blood flow, white matter lesions and brain atrophy: the SMART-MR study. J Cereb Blood Flow Metab. 2008;28(3):633-9.

14 Van Der Veen PH, Muller M, Vincken KL, Hendrikse J, Mali WP, Van Der Graaf Y, et al. Longitudinal relationship between cerebral small-vessel disease and cerebral blood flow: the second manifestations of arterial diseasemagnetic resonance study. Stroke. 2015; 46(5):1233-8.
15 Bisschops RH, Van Der Graaf Y, Mali WP, Van Der Grond J. High total cerebral blood flow is associated with a decrease of white matter lesions. J Neurol. 2004;251(12):14815.

16 Hashimoto T, Yokota C, Koshino K, Shimomura R, Hino T, Moriguchi T, et al. Cerebral blood flow and metabolism associated with cerebral microbleeds in small vessel disease. Ann Nucl Med. 2016;30(7):494-500.

17 Shi Y, Thrippleton MJ, Blair GW, Dickie DA, Marshall I, Hamilton I, et al. Small vessel disease is associated with altered cerebrovascular pulsatility but not resting cerebral blood flow; 2018.

18 Nezu T, Yokota C, Uehara T, Yamauchi M, Fukushima K, Toyoda K, et al. Preserved acetazolamide reactivity in lacunar patients with severe white-matter lesions: 15O-labeled gas and $\mathrm{H} 2 \mathrm{O}$ positron emission tomography studies. J Cereb Blood Flow Metab. 2012; 32(5):844-50.

19 Wong SM, Jansen JFA, Zhang CE, Hoff EI, Staals J, van Oostenbrugge RJ, et al. Bloodbrain barrier impairment and hypoperfusion are linked in cerebral small vessel disease. Neurology. 2019;92(15):e1669-77.

20 Østergaard L, Granfeldt A, Secher N, Tietze A, Iversen NK, Jensen MS, et al. Microcirculatory dysfunction and tissue oxygenation in critical illness. Acta Anaesthesiol Scand. 2015 59(10):1246-59. 